\title{
NIVELES DE ESTRÉS Y PATRONES DE ADAPTACIÓN DE MUJERES DIAGNOSTICADAS CON CÁNCER DE MAMA DEL ÁREA METROPOLITANA DE SAN JUAN, A MAYO DE 1998
}

\author{
María M. Lizardi de Fernández*
}

\section{Planteamiento del Problema y Justificación}

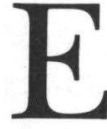

$\mathrm{n}$ la actualidad el trabajador social tiene un lugar en el equipo de profesionales de la salud. Una de las enfermedades que más aqueja a la sociedad puertorriqueña es el cáncer. Entre los tipos de cáncer uno de los que más se destaca es el cáncer de mama.

El cáncer de mama es la forma más común de cáncer en la mujer norteamericana. Una de cada nueve de éstas desarrollará cáncer de mama durante su vida. Por razones desconocidas la incidencia de cáncer de mama en la mujer norteamericana va en aumento (Anderson, 1997). Para el año 1993 murieron 290 mujeres a causa del cáncer de mama (Puerto Rico, Departamento de Salud, 1995). Sin embargo, este tipo de cáncer es uno que detectado a tiempo ofrece mayores oportunidades de supervivencia. Los adelantos médicos permiten detectar hoy en día la enfermedad en sus comienzos lo que alarga la expectativa de vida.

Se han llevado a cabo muchos estudios científicos sobre el cáncer. No obstante, la literatura revisada da énfasis a los aspectos médicos. Los aspectos socio-emocionales se han visto rezagados en

\footnotetext{
* Catedrática, Escuela Graduada de Trabajo Social, Universidad de Puerto Rico
} 
la literatura actual sobre el cáncer en comparación a los aspectos médicos. Sin embargo, se observa en la actualidad que la influencia de factores psicosociales en el desarrollo del cáncer y en su tratamiento ha dejado de ser un tópico de especulación para convertirse en un creciente interés científico (Peteet, 1986).

Las personas que experimentan enfermedades que amenazan su expectativa de vida reaccionan con pánico, dolor y confusión. Es importante que éstas reciban una comunicación clara y directa para que así puedan tomar decisiones respecto a su tratamiento y modificaciones si algunas, en su estilo de vida. Muchas veces el personal médico ignora los procesos básicos de la consejería para lidiar con la angustia y temor que provoca el diagnóstico de una enfermedad terminal como lo es el cáncer. Es en esos momentos que el trabajador social, psicólogo o consejero entra en la escena. El profesional de la conducta humana apoya al individuo diagnosticado para que entienda, explore y escoja entre las alternativas de tratamiento que se le presentan.

Al ser diagnosticada con cáncer de mama la mujer experimenta niveles altos de estrés. No solamente ésta se siente atemorizada por la amenaza a su expectativa de vida, sino que experimenta confusión en torno a los procedimientos de tratamiento. Las mamas aunque no constituyen un órgano vital para la persona, tienen un significado subjetivo para cada mujer. Es una parte del cuerpo a la cual se le da importancia y para muchas representa su capacidad reproductiva, en femineidad o un atributo de belleza. Todos estos significados varían de mujer a mujer y deben ser considerados en el momento de explicar el tipo de tratamiento a recomendar. El personal médico necesita ser sensible a las necesidades de la paciente y darle importancia a los significados psicológicos de ésta.

La autora del presente estudio es paciente de cáncer de mama. Este hecho contribuyó a aumentar su interés por estudiar esta enfermedad. Toda experiencia de vida constituye un proceso de aprendizaje. Pero a menudo dichos procesos conllevan niveles de estrés y requieren de sistemas de apoyo formales e informales que faciliten los mecanismos de adaptación psico-social.

Díaz-Estrada (1989) expone la importancia del consejero en la recuperación del paciente con cáncer debido a que los médicos enfocan mayormente el aspecto clínico y carecen del adiestramiento especializado y necesario para lidiar con la conducta humana. Señala además, que el modelo de consejería debe incluir al grupo 
familiar para promover una mejor adaptación dentro de dicho sistema. El trabajador social que labora en escenarios médicos al igual que el consejero necesita ampliar los conocimientos que le permitan desempeñar un rol competente dentro del equipo interdisciplinario que atiende a las mujeres que padecen de cáncer de mama.

Existe la necesidad de ampliar la literatura en trabajo social en el área de cáncer de mama, especialmente considerando los aspectos psico-sociales. Dentro de estos aspectos se destaca el estrés y sus efectos en la paciente de cáncer de mama. El presente estudio explora los niveles de estrés así como los patrones de adaptación asociados a la mujer que sufre cáncer de mama. Se espera que con el presente estudio se amplíen las estrategias de intervención con esta población.

\section{Marco Conceptual}

Toda investigación debe estar enmarcada en un marco conceptual que dirija los trabajos. Para el presente estudio la autora seleccionó la Perspectiva Ecológica expuesta por Germain y Gitterman (1980). La Perspectiva Ecológica es holística y transaccional. Enfoca el grado en que la unidad persona-ambiente se entrelaza y en los intercambios o transacciones recíprocos entre la gente y el ambiente, el cual puede apoyar o inhibir el impulso de adaptación. Esta perspectiva integra el tratamiento y la reforma mediante la conceptualización y el énfasis en las transacciones entre la gente y su ambiente físico y social (Miranda, 1990). La adaptación es un proceso activo y recíproco donde el ser humano y el ambiente se influyen mutuamente. También se ofrece atención tanto a los factores del individuo como a factores externos en su entorno (Germain y Gitterman, 1980).

La perspectiva ecológica dirige la intervención hacia un método de práctica integrado que da énfasis a las fortalezas de las personas y hacia la remoción de obstáculos ambientales que limitan su desarrollo (Miranda, 1990). Este marco conceptual también da énfasis a los sistemas de apoyo formales e informales al explicar el funcionamiento de las personas. Reconoce que la persona también puede estar expuesta al estrés del ambiente el cual si llega a un grado mayor que lo que la persona puede manejar, podría exponer a ésta a un estado de crisis. Se visualiza la crisis como un proceso que no 
necesariamente lleva a un deterioro de la persona sino que podría resultar en crecimiento de ésta si se cuenta con los recursos internos y externos apropiados para su manejo.

\section{Revisión de la literatura}

El cáncer es una enfermedad o un grupo de enfermedades caracterizada por un crecimiento anormal de las células. Este crecimiento no controlado y desordenado de células puede expandirse y si no se detiene podría resultar en la muerte del que lo padece (Conti, 1989). El término cáncer significa "el cangrejo" y proviene del griego. A través de la historia, la enfermedad ha sido visualizada como una sentencia de muerte, sin embargo en los últimos años esta imagen ha empezado a desaparecer (Taché, 1986). El cáncer puede atacar cualquier órgano del cuerpo. Existen más de 250 tipos diferentes de cáncer (Conti, 1989).

El cáncer de mama es muy común en la mujer aunque puede ocurrir en el hombre en menor proporción. Los síntomas que se observan en las mamas incluyen entre otros, endurecimientos palpables, hinchazón, cambio en la forma de los pezones, irritación en la piel y descarga de los pezones. El uso de la mamografía puede detectar tumores en una etapa temprana que no presenta ninguno de los síntomas antes mencionados. Por esta razón se recomiendan los exámenes manuales de las mamas a partir de los 20 años. A partir de los 40 años la sociedad americana del cáncer recomienda que se lleve a cabo una mamografía anualmente.

El tratamiento del cáncer de mama puede incluir cirugía. Ésta puede ser una lumpectomía o remoción del tumor y tejido adyacente, remoción de la mama o mastectomía, remoción de la mama y nódulos linfáticos (mastectomía radical modificada) o la remoción del seno, los nódulos linfáticos y los músculos subyacentes o mactectomía radical.

De acuerdo al cuadro clínico que presente la paciente, las pruebas de patología y de laboratorio, el médico puede recomendar el tratamiento apropiado. Parte del tratamiento podría incluir radioterapia, quimioterapia y el uso de tamoxifen. La droga tamoxifen no es recomendable para todas las mujeres y el médico es el indicado para recomendar su uso. Esta droga tiene el efecto de bloquear el estrógeno en el seno de la mujer. El estrógeno ha sido asociado al cáncer del seno. 
El concepto de estrés fue formulado por primera vez por Hans Selye en 1936 (Selye, 1986). Se refiere a la respuesta no específica del cuerpo a cualquier demanda. Es un proceso de adaptación que se desarrolla como una reacción a un estímulo llamado estresor y se manifiesta a través de cambios en los niveles hormonales y en el tamaño de los órganos (Taché, 1986).

Selye (1986) propone que cada estresor actúa diferente en cada persona. El efecto del estrés en los órganos dependerá en gran medida de la condición de éstos. El estrés acompaña todas las enfermedades y todas las actividades en la vida pero cuando el organismo se expone a un nivel de estrés incompatible con sus posibilidades de resistencia o adaptación adecuadas al estrés, produce enfermedad (Selye, 1986).

El rol del estrés como precursor del cáncer ha sido ampliamente estudiado pero aún no está claro. El estrés y el cáncer están relacionados de acuerdo a Selye (1986) en tres formas. El cáncer puede producir considerable estrés, el estrés puede agravar el cáncer y el estrés puede inhibir o prevenir el cáncer.

Ya para el 1759, Sir Richard Guy exploraba la idea de que el cáncer de mama era predominante en mujeres que mostraban histeria y melancolía. En dos de los casos que éste estudió describió la incidencia de cáncer de las mamas en mujeres que habían experimentado la muerte de un hijo o encarcelamiento. En el 1885, Willard Parker eminente médico norteamericano observó que la angustia era frecuente en la historia inmediata de pacientes de cáncer de mama. En 1887 Cutter señaló que la depresión mental es un elemento que no debe ignorarse en los pacientes de cáncer. Otros investigadores en la actualidad han hecho observaciones similares a estas ideas planteadas en los siglos 18 y 19 (Selye, 1986). Entre los aspectos se encuentran: 1) la pérdida de una figura significativa, 2) frustración ante situaciones y metas en la vida y 3 ) tendencia hacia la desesperación, angustia y desesperanza al enfrentar el estrés, frustraciones o pérdidas. Aunque ninguna de éstas se ha probado, definitivamente se consideran elementos de predisposición a la carcinogénesis.

Benjamín (1987) plantea que el estrés que resulta de un estresor o evento negativo tiende a deprimir el sistema inmunológico. Las respuestas de adaptación del cuerpo incluyen aumento en los latidos del corazón flujo de adrenalina y otras hormonas en el torrente sanguíneo. Algunas de estas hormonas pueden contribuir 
a deprimir el sistema inmunológico. Si el sistema inmunológico no funciona adecuadamente por un período extenso de tiempo estará incapacitado para combatir células cancerosas que se encuentran en el organismo. Este permite que dichas células se unan y formen un tumor canceroso. Si se utiliza mucha energía para el manejo de procesos o problemas mentales y emocionales habrá menos energía disponible para manejar problemas físicos.

El cáncer de mama es una enfermedad con implicaciones psicosociales para la mujer que lo padece. Parrilla-Sotomayor (1986) estudió el impacto de la mastectomía en el auto-concepto de un grupo de mujeres en etapa post-operatoria. El estudio destaca que para la mujer con mastectomía, la malignidad y la cirugía tiene un significado real y simbólico que a menudo ocasiona respuestas cargadas de tensión. Para la mujer con cáncer de mama, la localización de su aflicción es significativa debido al significado real y simbólico que tienen las mamas en la sociedad occidental. Los hallazgos del estudio sugieren que la mastectomía es un evento traumático que ejerce influencia en la auto-percepción de la mujer que la experimenta. La mujer con mastectomía obtuvo puntuaciones más bajas de auto-concepto al compararlas con un grupo de mujeres sanas de la misma edad.

Vega-Franki (1992) en un estudio con pacientes de cáncer de mama y próstata analizó el ajuste psicosocial de la condición de una muestra de 46 pacientes, provenientes del Hospital Oncológico Dr. Isaac González Martínez. De éstos, 30 eran mujeres con cáncer de mama y 16 eran hombres con cáncer de próstata. Además de medir el ajuste psico-social hacia el cáncer, se consideraban otras variables personales: sexo, edad, ocupación, escolaridad, estado civil, número de hijos, zona de residencia y estado socio-económico. Entre los hallazgos principales se encontró que los hombres fueron los que más problemas de ajuste presentaron, especialmente en áreas como el ámbito vocacional, relaciones sexuales y aspectos económicos. Las mujeres demostraron que tienen mejor ajuste psicosocial en todas estas áreas. $\mathrm{El}$ autor concluyó que un diagnóstico de cáncer afectaba el balance del individuo y del grupo social.

Los sobrevivientes de cáncer muchas veces cargan con estigmas sociales, según buscan redefinirse a si mismos en un ambiente que no está preparado para el cuidado de la salud. Las reacciones de los pacientes de cáncer a su diagnóstico y a los síntomas tanto como su estilo de adaptación a la enfermedad está basada en 
variables personales, sociales y culturales. En el manejo de la enfermedad es importante incorporar a la familia del paciente con cáncer, ya que ésta constituye el principal sistema de apoyo psicosocial del paciente. Tanto el paciente como la familia necesitan apoyo psicosocial para enfrentarse al impacto del cáncer en sus vidas diarias.

Es importante tomar en consideración la familia del paciente con cáncer para el manejo de la enfermedad, ya que constituye el principal sistema psicosocial en el que se mueve el paciente. Se ha encontrado que los disturbios psicológicos son mayores cuando las respuestas de los pacientes con cáncer han mostrado fatalismo, desplazamiento o proyección.

Otros hallazgos de Vega Franki fueron los siguientes:

- El $81 \%$ de los pacientes presentaron un estado de ánimo de optimismo hacia la condición. En el área vocacional las mujeres tuvieron mejor ajuste que el hombre. El hombre con cáncer de próstata experimentó mayor desajuste que la mujer con cáncer de mama. Los hombres presentaron cambios en sus objetivos de trabajo y conflictos de convivencia con sus compañeros de labores.

- Tanto en hombres como en las mujeres las relaciones conyugales no se afectaron significativamente. En el aspecto económico los sujetos experimentaron crisis económica desde leve hasta grave. El apoyo familiar tanto de la familia nuclear como de la extendida representó apoyo adecuado para el desarrollo positivo de las actividades cotidianas, en la comunicación afectiva y en la comunicación general.

- Los pacientes experimentaron incapacidad física desde leve hasta moderada, siendo los hombres los más afectados.

- El ambiente social no se afectó significativamente, mayormente porque la actividad social de los pacientes estaba limitada en muchos aspectos debido a la edad. Los hombres se afectaron mentalmente más que las mujeres, aunque la mayoría de los sujetos estudiados no demostraban haber sido afectados. Los pacientes demostraron tener un buen ajuste psico-social a la condición. La edad representó un papel muy importante en el ajuste, según quedó demostrado en los hallazgos. Otra variable fue el género, ya que quedó demostrado que la mujer tiene mejor ajuste psicosocial que los hombres. 
Lieberman (1982) estudió el rol de los sistemas de apoyo en respuesta al estrés. En su análisis Lieberman (1982) señala que el estrés se visualiza como el producto de ciertos eventos o condiciones en la vida de las personas que lleva a una variedad de consecuencias. Entre dichas consecuencias se encuentra esfuerzos de lidiar con la situación, estrategias de defensa, sentimientos de distrés y alteración de la calidad del funcionamiento de los roles sociales principales y síntomas psicológicos y fisiológicos. Uno de los aspectos estudiados sobre los sistemas de apoyo como estrategia para lidiar con el estrés es el pedir ayuda. La revisión de literatura revela que la mayoría de las personas buscan ayuda ante eventos de gran estrés. Dos de los factores que establecen la diferencia entre los que buscan ayuda y los que no buscan ayuda son la edad y la raza. Las personas que buscan ayuda desean obtener un sentido de apoyo y guía ante su situación. Inicialmente acuden a la familia y a las amistades, las agencias de servicio son contactados como último recurso. La mayor parte de las personas estudiadas reflejó que perciben a su red social como una fuente de ayuda. En muchas circunstancias las personas acuden a agencias profesionales sólo cuando la ayuda no está disponible en su red social (Lieberman, 1982).

Goldberg and Tull (1983) exponen la importancia del apoyo social en la adaptación psicosocial al cáncer. Estos señalan que para las personas el amor y las relaciones de apoyo proveen la fuerza necesaria para enfrentarse a los retos de la vida y la muerte. El apoyo social también contribuye a lidiar con el distrés psicológico y facilita los mecanismos de adaptación y enfrentamiento a la enfermedad. Estos autores destacan el concepto de persona significativa clave. Dicha persona puede ser la pareja, un hijo(a) $u$ otro familiar o persona cercana al paciente. Como sistema de apoyo esta persona tiene unas funciones claves. Entre estas funciones estan el ayudar a mantener el sistema de apoyo del paciente, ayudar a que el(la) paciente obtenga un sentido de autonomía, servir de intercesor por el(la) paciente cuando fuera necesario, promover la comunicación entre el(la) paciente y su familia y amistades y facilitar la expresión de sentimientos.

El presente estudio exploró el impacto del cáncer de mama en la mujer que lo padece desde su perspectiva individual y subjetiva. Se da énfasis a los sistemas de apoyo como alternativas para lidiar con el estrés producido por la enfermedad. 


\section{Propósitos del estudio}

El propósito general de este estudio fue describir los niveles de estrés y patrones de adaptación psicológicos y sociales de mujeres diagnosticadas con cáncer de mama.

Los propósitos específicos son:

1. Explorar la naturaleza del proceso de información y orientación que recibió la paciente.

2. Describir el impacto de su condición en su funcionamiento social.

3. Conocer los patrones de adaptación psico-sociales de la paciente.

4. Determinar el nivel de estrés experimentado por la paciente después del diagnóstico de cáncer.

5. Describir la percepción de la mujer con cáncer de mama sobre el manejo de su condición por parte de los sistemas de apoyo formales e informales.

\section{Preguntas de investigación}

Con el propósito de guiar los trabajos del presente estudio la autora desarrolló las siguientes preguntas:

1. ¿Cómo fue el proceso de información y orientación que recibió la paciente?

2. ¿Cómo percibía el nivel de estrés la mujer diagnosticada con cáncer de mama después del diagnóstico?

3. ¿Cómo ha impactado la enfermedad el funcionamiento social de la mujer?

4. ¿Cuáles eran las estrategias de manejo de estrés de la mujer con cáncer de mama?

5. ¿Qué sistemas de apoyo formales e informales tiene la mujer con diagnóstico de cáncer de mama?

6. ¿Cómo es la percepción de la mujer con cáncer de mama sobre el manejo de su condición por parte de los sistemas de apoyo formales e informales? 


\section{Diseño metodológico}

\section{Tipo de Diseño}

En este estudio el diseño utilizado fue descriptivo ubicado en una sola celda y en un solo tiempo, el presente. El género testimonial se integró al diseño de este estudio para proveer así a las participantes la oportunidad de brindar sus vivencias y experiencias así como sus opiniones sobre los servicios recibidos.

\section{Descripción de la población y selección de la muestra}

La población de este estudio estuvo constituida por 20 mujeres entre las edades de 46-70 años residentes de Puerto Rico. La muestra fue no probabilística seleccionada por su disponibilidad para participar en el estudio. Para llegar a la población se realizaron visitas a centros de tratamiento para pacientes de cáncer de mama.

\section{Instrumento de Observación}

Para el siguiente estudio la autora elaboró una guía de preguntas que sirvió para dirigir la entrevista y el testimonio que ofrecieron las participantes del estudio. Mediante ésta se pretendió recoger los datos pertinentes de las variables bajo estudio. Con el fin de facilitar el proceso de la entrevista la autora diseñó las preguntas para que la misma fluyera en forma de diálogo.

La guía constó de tres partes. La primera parte consistió de preguntas sobre aspectos socio-demográficos de las mujeres participantes. La segunda parte recogía información sobre los niveles de estrés experimentados por éstas. La cuarta parte expone los patrones de adaptación psico-social.

\section{HALLAZGOS}

\section{Aspectos socio-demográficos}

Las edades de las mujeres entrevistadas fluctuaron entre los 46 y los 70 años de edad. Solamente dos de las mujeres entrevistadas vivían solas. El resto vivía con el esposo, hijos y otros familiares. Este aspecto resultó importante en el proceso de adaptación a la enfermedad y el estrés resultante. El convivir con otras personas significó para las mujeres entrevistadas un apoyo para lidiar con su enfermedad. 
Los hallazgos obtenidos indican que los sujetos bajo estudio residían en diversos pueblos de la Isla. Sin embargo, el por ciento mayor de los sujetos residía en San Juan u otros pueblos del área metropolitana.

La autora encontró que había diversidad en las preferencias religiosas de las mujeres participantes del estudio aunque el $50 \%$ eran católicas. Se consideró el explorar sobre esta área ya que se interesaba conocer si la preferencia religiosa constituirá un sistema de apoyo para las mujeres entrevistadas.

Numéricamente las mujeres casadas constituían el por ciento mayor de las entrevistadas con un 40 por ciento, estas eran seguidas por viudas con un 35 por ciento y divorciadas con un 25 por ciento.

En relación al status de empleo se encontró que quince de las mujeres entrevistadas eran amas de casa. De las cinco que estaban trabajando una era maestra, una trabajaba para el gobierno federal y tres ocupaban posiciones técnicas en la empresa privada.

Un $55 \%$ de las mujeres tenían de uno a tres hijos un $45 \%$ tenía de cuatro a seis hijos y un cinco por ciento tenía 6 hijos o más. Las edades de los hijos fluctuaban entre veinte y cuarenta y cinco años.

Once de las mujeres entrevistadas fueron madres en el intervalo de veinte a treinta años, lo que constituye un cincuenta y cinco por ciento de la muestra. Ocho de ellas tuvieron sus hijos después de los treinta años de edad constituyendo un cuarenta por ciento y solamente una de éstas tuvo su primer hijo antes de los veinte años. Solamente una de las mujeres entrevistadas señaló haber lactado a sus hijos.

Al explorar sobre la familia de orientación se encontró que quince de las mujeres entrevistadas o el setenta y cinco por ciento de ellas tenían de dos a cuatro hermanos. Cuatro de ellas o el veinte por ciento de la muestra tenía más de cuatro, solamente una tenía un solo hermano o el cinco por ciento de la muestra.

\section{Niveles de estrés experimentado}

Al explorar sobre el nivel de estrés experimentado por las mujeres participantes del estudio se formularon varias preguntas relacionadas al diagnóstico de la enfermedad, y al proceso de información utilizado para dar a conocer el mismo. Al explorar sobre los síntomas observados se encontró que el por ciento mayor de las mujeres $(50 \%)$ descubrieron su enfermedad al palpar un 
abultamiento en o cerca de los senos o en la zona debajo de los brazos.

A continuación se ilustra éste y otros datos relacionados con los síntomas experimentados por las mujeres entrevistadas.

Síntoma o Forma de Diagnóstico

Abultamiento o engrosamiento en o cerca de los senos o en la zona debajo de los brazos

Microcalcificación descubierta por mamografía

Descarga de sangre por el pezón o piel escamosa en el pezón o la aréola

Cambio anormal en el tamaño o en la forma de los senos (hinchazón)

Cambio en el color o la textura de la piel de los senos (huequitos, fruncimientos, engrosamiento, escamosidad o colores rojizos)
Frecuencia Por Ciento

Total $20 \quad 100.0$

$10 \quad 50.0$

$5 \quad 25.0$

$2 \quad 10.0$

2

10.0

1

5.0

Cabe destacar que un veinticinco por ciento de las mujeres entrevistadas no habían experimentado ningún síntoma físico sin embargo descubrieron su enfermedad a través de la mamografía. De esto se desprende la importancia de dicho método de diagnóstico.

\section{Método de Información del Diagnóstico}

La gran mayoría de las mujeres entrevistadas (95\%) fueron informadas de su condición por el médico en su oficina. Solamente una de estas fue informada por teléfono. Un 75 por ciento de las mujeres estaban acompañadas al ser informadas de su diagnóstico por el médico. De éstas el 45 por ciento estuvieron acompañadas por algún familiar o amistad cercana y 30 por ciento recibió la noticia estando su marido presente.

\section{Sentimientos Experimentados al conocer el Diagnóstico}

$\mathrm{Al}$ explorar con las mujeres participantes sobre los sentimientos experimentados imperaron los sentimientos de tristeza, confusión, 
enojo y temor manifestado por un $95 \%$ de estas, solamente una de las participantes expresó haberse sentido cómoda y optimista.

\section{Familiares con Cáncer de Seno}

El 40 por ciento de las entrevistadas tenían familiares que habían padecido o padecían cáncer de seno. Quince por ciento señalaron a sus hermanas $10 \%$ a sus madres $10 \%$ a tías y $5 \%$ a una hija.

\section{Tratamiento recibido}

De explorar sobre el tipo de tratamiento recibido por las mujeres éstas señalaron las siguientes combinaciones de los mismos como se expresa en la siguiente distribución por frecuencia.

Tipo de tratamiento

Radioterapia

Tlamoxifen (Nolvadex)

Mastectomía

Lumpectomía

Quimioterapia

Medicina arternativa
Frecuencia de Mención

Es interesante observar que el número de mujeres que se sometieron a la mastectomía o remoción quirúrgica del seno fue similar a las que se sometieron a la lumpectomía combinada con otros métodos de tratamiento. También es digno de notar que una de las participantes optó por la medicina alternativa luego de haber recibido los resultados de la biopsia. Esta señaló que este tipo de tratamiento incluía una dieta especial, ejercicios, masajes y meditación.

Nivel de Estrés percibido y estrategias de manejo

Noventa por ciento de las mujeres entrevistadas expresaron que el nivel de estrés experimentado en su vida fue mayor luego de recibir el diagnóstico de cáncer de seno. Las preocupaciones primordiales de las mujeres entrevistadas fueron el temor a una muerte prematura, el temor a la mutilación y el impacto en la familia. Estos y otros datos se ilustran a continuación: 
Preocupación percibida

Muerte

Temor a la mutilación

Impacto en la familia

Sufrir dolor o complicaciones

Situación económica familiar

Efectos en el trabajo

Transporte para recibir tratamiento

Ausencia de seguro médico
Frecuencia de Mención

15

9

7

6

5

3

3

\section{Estrategias de Manejo de Estrés}

De explorar sobre las estrategias de manejo de estrés se encontró que la alternativa mencionada con mayor frecuencia fue la utilización de la meditación y la oración basada en la fe religiosa. Otras estrategias de manejo de estrés mencionadas fueron las siguientes:

Estrategia de manejo de estrés

Frecuencia de Mención

Oración/meditación 20

Lectura de libros de autoayuda

Cambios en la dieta

Ejercicios físicos

Compartir en familia

\section{Impacto de la enfermedad en el funcionamiento social}

\section{Impacto Personal}

Para explorar esta área se hicieron preguntas relacionadas a la vida personal, familiar, en el empleo y en la comunidad. Un 100\% de las mujeres entrevistadas expresaron que de alguna manera el cáncer había impactado sus vidas positiva o negativamente.

Una de las participantes expresó: "No puedo entender mis sentimientos. El primer impacto fue pensar en la muerte. Luego recapacité busqué ayuda en mi familia, en los hermanos de la iglesia. Ellos me ayudaron a visualizar la situación de manera diferente". 
Otra participante señaló: "Lo que más me ha afectado son los efectos de la quimioterapia. A veces me siento muy mal, nauseas, vómitos, tú sabes, terrible. Pero trato de seguir adelante por mi familia".

Todas las entrevistadas que recibieron tratamiento de quimioterapia mostraron preocupación por los cambios físicos especialmente la caída del cabello. Para lidiar con esto utilizaban pelucas, pañuelos y sombreros. Sin embargo, es interesante ver que ninguna de ellas planeó estas estrategias antes del tratamiento sino después de haber iniciado el mismo.

De las 10 participantes que habían optado por la mastectomía solamente una expresó no sentir ningún impacto por esta operación.

"A mi no me importó perder el seno, era peor perder la vida. Yo quiero vivir. Además, ya eso dio lo que iba a dar. Cuando el médico me dio las alternativas yo opté por ésta. Como mis nódulos salieron negativos no tuve que someterme, ni a radioterapia, ni a quimioterapia. Solamente uso la "Nolvadex".

\section{Impacto en el hogar}

Cuando un miembro de la familia tiene cáncer todos sus integrantes sufren el impacto. Así fue señalado por 18 de las 20 mujeres entrevistadas. Las dos mujeres que señalaron que no hubo impacto en la familia vivían solas. El tipo de impacto señalado fue diverso destacándose un efecto positivo. Catorce de las participantes señaló que los familiares tenían un mayor acercamiento con ellas, expresando amor y preocupación. El diagnóstico de cáncer conllevó unos cambios en la distribución de las tareas en el hogar lo cual fue aceptado por los miembros de la familia.

Dos de las entrevistadas destacaron que el estrés causado por la enfermedad había provocado discusiones entre los miembros de la familia. Esto se relacionaba también a los ajustes que había que hacer en términos de las tareas del hogar. Una de las participantes señaló: “A veces los muchachos discuten entre sí porque unos cooperan más que los otros. A veces muestran resentimiento porque no podemos llevar a cabo actividades que anteriormente hacíamos".

Otra expresó que a veces discutía con su esposo porque la "trataba como si ella no pudiera hacer nada". "Eso me molesta porque me gusta valerme por mí misma”.

Dos de las participantes señalaron una reacción de aislamiento por parte de los miembros de la familia extendida. Esto puede 
entenderse como un mecanismo para lidiar con la ansiedad y el temor que provoca el diagnóstico de cáncer. A continuación se expresan numéricamente estos datos.

Funcionamiento en el hogar

Total

Mayor acercamiento de los miembros

Discusiones

Aislamiento

No hubo impacto
Frecuencia Por Ciento

20

14

100.0

70.0

10.0

10.0

2

10.0

\section{Funcionamiento en el empleo}

De las cinco participantes que estaban empleadas, tres de éstas expresaron que la enfermedad había tenido un impacto en el empleo debido a la frecuencia de los tratamientos y sus efectos secundarios. Como alternativa a esto, los patronos hicieron ajustes en las tareas asignadas. Una de las participantes señaló que había agotado todas sus vacaciones por enfermedad y regulares debido a los tratamientos. También presentó una alternativa que practicaban en su agencia llamada "donar días", de esta manera en circunstancias especiales un empleado podía donar días de vacaciones regulares a otro empleado. Ella tuvo esta oportunidad.

\section{Funcionamiento en la comunidad}

El 90 por ciento de las participantes entrevistadas manifestó que en términos de las relaciones con la comunidad no habían experimentado muchos cambios al compararlo con el impacto en la vida personal y familiar. Estas expresaron que la enfermedad las había hecho estar más conscientes del amor por su familia y establecieron la convivencia familiar como prioridad. Las participantes que recibían tratamiento de quimioterapia expresaron que su participación en actividades sociales había disminuido debido a los efectos del tratamiento. Sin embargo, mencionaron que los vecinos y los amigos habían mostrado mayor acercamiento hacia éstas una vez conocido el diagnóstico. 


\section{Apoyo Psico-Social Recibido}

Al explorar con las participantes sobre el apoyo recibido en términos psico-sociales se destacó el rol de la familia, tanto la de origen como la de procreación así como las amistades. Las participantes también destacaron el rol del médico y del trabajador social. Al indagar sobre el tipo de apoyo recibido las 10 participantes que habían optado por la mastectomía informaron que recibieron orientación de la trabajadora social del hospital sobre la prótesis, donde obtenerla y asuntos relacionados al plan médico. En ninguno de los casos se indagó o intervino con las participantes en términos de los efectos emocionales de la mastectomía en la mujer. Sin embargo, se catalogó la relación profesional como positiva. Esto también se observó en la relación de los médicos que fueron mencionados por 12 de las participantes. Estas percibieron a sus médicos como cariñosos, interesados por su bienestar y atentos aunque no abordaran el tema de los aspectos emocionales de la enfermedad. Dos de las participantes señalaron que tuvieron que buscar ayuda de un psiquiatra para lidiar con su situación.

La iglesia como sistema de apoyo fue mencionada por 10 de las participantes.

- "Los hermanos de la iglesia nunca me abandonaron. Me visitaron al hospital y en mi casa".

- "La oración me ha ayudado mucho a manejar el dolor que esta enfermedad me ha causado".

- "Si no fuera por mi Cristo ya me hubiera muerto".

- "Yo sé que Dios es leal y que me sanará".

A continuación se presenta un desglose de estos datos.

Fuentes de apoyo emocional

Amistades

Frecuencia de Mención

Familia de procreación

18

Familia de origen

18

Médico

15

Iglesia

12

Trabajador Social

10

Grupos de apoyo "Yo Puedo"

Psiquiatría 
Cabe destacar que solamente seis de las participantes recibieron ayuda específica para lidiar con los aspectos psico-sociales de la enfermedad. Dos de éstas buscaron ayuda en un psiquiatra.

"El me ayudó a ver la situación de manera diferente. Yo asociaba el cáncer con la muerte y ahora lo veo como una oportunidad de apreciar y celebrar la vida. No me arrepiento de haber buscado ayuda psicológica. A veces los amigos y familiares desean ayudarte pero no están capacitados para hacerlo. Recuerdo una vez que alguien me dijo que Dios me había mandado esa prueba y sentí muchísimo coraje. También me daba coraje cuando me decían que no me preocupara porque yo sé que tengo que preocuparme para poder sanarme. Ahora me siento más tranquila, vivo en el presente y no pienso en la muerte tanto como antes. Definitivamente yo le recomiendo a otras personas que estén pasando por una situación familiar que busquen ayuda profesional".

Las entrevistadas que participaban del grupo de apoyo "Yo Puedo" el cual es auspiciado por el Hospital del Maestro expresaron satisfacción con la experiencia. El grupo les proveyó de información sobre la enfermedad, conocieron nuevas amistades y tenían un entretenimiento nuevo. Todas ellas expresaron que el grupo les enseñó a percibir la enfermedad de manera diferente y a comprender que no estaban solas en ese proceso.

\section{Fortalezas Percibidas}

En términos de las fortalezas que las participantes percibían en ellas como personas se destacaron las siguientes respuestas.

\section{Fortalezas}

Fe religiosa

Educación / Conocimiento

Optimismo y sentido de humor
Frecuencia

20

15

3

2

\section{Por Ciento}

100.0

75.0

15.0

10.0

La gran mayoría de las participantes señaló que su mayor fortaleza era la fe, el practicar una creencia religiosa les daba un sentido de esperanza en la vida. También proveía una explicación para la vida y la muerte. Tres de las participantes señalaron que el tener información clara sobre su condición era su mayor fortaleza. Dos 
de las participantes señalaron su optimismo y sentido de humor como sus mayores fortalezas.

\section{Conclusiones y recomendaciones}

Para la realización del presente estudio la autora formuló cinco preguntas de investigación las cuales se discuten a continuación:

\section{1. ¿Cómo percibía el nivel de estrés la mujer diagnosticada con cáncer del seno o mama después del diagnóstico?}

La gran mayoría de las mujeres entrevistadas (90\%) expresaron que el nivel de estrés experimentado en su vida fue mayor luego de recibir el diagnóstico. Las preocupaciones primordiales de éstas fueron el temor a una muerte prematura, el temor a la mutilación y el impacto de la enfermedad en la familia. De acuerdo a la literatura revisada el estrés puede afectar el sistema inmunológico de la persona haciéndola vulnerable a otras enfermedades y afectando el proceso de recuperación. Por tal razón la mujer necesita ayuda para desarrollar estrategias efectivas en el manejo del estrés. Esto debe incorporarse al tratamiento médico recibido. La ayuda ofrecida a la mujer debe trascender el aspecto físico incluyendo los aspectos psicosociales. El tratamiento de la mujer debe verse de una forma holística dando énfasis a la interacción de sus sistemas cognoscitivo, afectivo, somático, interpersonal y conductual. Los familiares deben incorporarse al tratamiento.

\section{2. ¿Cómo ha impactado la enfermedad el funcionamiento so-} cial de la mujer?

Al analizar el funcionamiento social se exploró sobre el impacto como persona, como miembro de una familia, como miembro de una comunidad y como empleada. Todos estos aspectos fueron impactados por la enfermedad sin embargo las participantes dieron mayor importancia a los aspectos personales y familiares.

En el aspecto personal se destacó que las mujeres que habían recibido quimioterapia se preocupaban no solamente por los efectos del tratamiento, sino por los cambios físicos como la pérdida del cabello y la resequedad de la piel. Las que además de recibir quimioterapia se habían sometido a la mastectomía albergaban un sentimiento de pérdida y mutilación. Solamente una de éstas señaló 
no haber recibido ningún impacto por la pérdida del seno. Ninguna de las mujeres que habían sido sometidas a la mastectomía había visualizado la alternativa de reconstrucción del seno.

En el ámbito familiar fue donde se detectó un impacto mayor, esto fue señalado por $90 \%$ de las participantes. Se concluye que el cáncer es una enfermedad con implicaciones en la familia. Por tal razón se recomienda que al tratamiento se integre la intervención con la familia especialmente con los esposos e hijos que se ven directamente afectados especialmente en una sociedad donde la mujer lleva prácticamente sola las riendas del hogar. La enfermedad conlleva el examinar y re-distribuir roles todo lo cual crea tensión en la familia. Por tal razón la familia debe recibir apoyo como grupo de manera que pueda hacer mejor utilización de los recursos y manejar los conflictos familiares. La intervención profesional en trabajo social debe ampliar para atender estas necesidades.

Otra de las áreas exploradas fue el impacto en el empleo. Solamente cinco de las participantes trabajaban. Todas ellas expresaron que sus patronos habían hecho ajustes en las tareas para responder a las necesidades de éstas. El aspecto que causaba mayor preocupación era las ausencias al empleo lo cual era menos tolerable en las participantes que trabajaban en el sector privado.

El funcionamiento en la comunidad fue visto como menos importante en comparación con el impacto personal y familiar. Aunque las participantes que recibían tratamiento de quimioterapia señalaron una disminución en las actividades sociales también destacaron un mayor acercamiento de vecinos y amigos una vez diagnosticada la enfermedad.

A la luz de estos hallazgos se recomienda que se puntualice más el rol del trabajador social en los escenarios que ofrecen tratamiento a mujeres con cáncer de mama. La intervención debe dirigirse a todas las pacientes y no solamente a las que optan por la mastectomía. Además la orientación no debe limitarme a conocer sobre las prótesis sino que debe ser más amplia y considerar los aspectos psico-sociales de la paciente.

\section{3. ¿Cuáles eran las estrategias de manejo de estrés de la mujer con cáncer de mama?}

Las estrategias de manejo de estrés mayor utilizadas estaban relacionadas a la vida religiosa de las participantes. La totalidad de éstas señaló la oración y la meditación como estrategia de manejo. 
Una de ellas manifestó: "Cuando oro hablo con Dios, cuando medito Dios habla conmigo". Es interesante que aunque practicaban diferentes creencias religiosas percibían la fe en un ser supremo como una fortaleza para sus vidas.

Aunque se reconoce la fe religiosa como una estrategia de manejo se observó la necesidad de fomentar otras estrategias de manejo de estrés. Esto requiere que estas personas y sus familias reciban orientación ya sea individual, familiar o grupal. Se recomienda que todos los hospitales organicen grupos de apoyo como el Hospital del Maestro que tiene el grupo "Yo Puedo". Las entrevistadas que habían participado en este grupo estaban mejor informadas y tenían una actitud más optimista para el manejo del estrés.

\section{4. ¿Qué sistemas de apoyo formales e informales tiene la mu- jer con cáncer de mama?}

Se encontró que las mujeres entrevistadas habían recibido apoyo de sistemas informales representados por la familia de origen y de procreación, las amistades y los vecinos. De los sistemas de apoyo formales se mencionaron los médicos, psiquiatras y trabajadores sociales. De todos ellos la familia ocupó el lugar de mayor importancia. Sin embargo, se concluye que aunque los familiares tenían buenas intenciones al ayudar a la paciente a veces carecían de la información necesaria y las destrezas para hacerlo. Esto nuevamente apunta a la necesidad de mayor orientación a la familia y un mayor énfasis en el rol del trabajador social.

5. ¿Cómo es la percepción de la mujer con cáncer de mama sobre le manejo de su condición por parte de los sistemas de apoyo formales e informales?

Se concluye que la mayoría de las entrevistadas está satisfecha con el manejo de su condición por los sistemas de apoyo formales e informales. Sin embargo, en algunos de los testimonios se desprende un sentimiento de insatisfacción por lo limitado que era el conocimiento sobre la enfermedad en familiares y amigos. En relación a los médicos se señaló que éstos daban mayor importancia al aspecto físico y muchas veces ignoraban los aspectos sociales y emocionales.

A base de estos hallazgos se concluye que existe la necesidad de ofrecer orientación y adiestramiento sobre los aspectos psico-sociales del cáncer para los profesionales en el área de la salud que trabajan en esta población. 


\section{Referencias}

Anderson, B.L. (1998). Stress and Immunity Breast Cáncer Project. Columbus: Ohio State University.

Benjamín, H.H. (1987). From Victim to Victor. Los Angeles: Jeremy P. Tarcher, Inc.

Conti, J. (1989). Counseling Persons with Cancer. Springfield: Charles C. Thomas Publishers.

Díaz Estrada, H. (1989). Los pacientes de cáncer y su familia: un modelo de consejería para la vida. Tesina de maestría inédita, Universidad de Puerto Rico, Recinto de Río Piedras, Programa Graduado de Orientación y Consejería.

Germain, C.B.; Gitterman, A. (1980). The Life Model of Social Work Practice. New York: Columbia University Press.

Goldberg, R. y Tull, R. (1983). The psychosocial dimensions of cancer: a practical guide for health care providers. New York: Free Press; London Collier MacMillan.

Guy, R. (1759). An Essay on Scirrhus Tumors and Cancers. London: J and A. Churchill.

Lieberman, M.A. (1982). The Effects of Social Supports on Responses to Stress. En: Golberger, L. y Breznitz S. Handbook of Stress: Theoretical and Clinical Aspects. New York: The Free Press.

Miranda, E. (1990). La práctica del trabajo social y el modelo ecológico. Manuscrito inédito. Universidad de Puerto Rico, Río Piedras: Escuela Graduadas de Trabajo Social.

Parker, W. (1885). Cancer: A Study of 397 Cases of Cancer of the Female Breast. New York: G.P. Putnam and Sons.

Parrilla Sotomayor, Ma. (1986). El impacto de la masectomía en el autoconcepto de un grupo de mujeres en etapa post-operatoria. Tesis de maestría inédita. Universidad de Puerto Rico, Recinto de Ciencias Médicas, Escuela Graduada de Salud Pública.

Peteet, J.R. (1986). Psychological Factors in the Causation and Course of Cancer. In Day, S.B. (Ed.). Cancer, Stress and Death. New York: Plenum Medical Book Company.

Puerto Rico (1995). Departamento de Salud. Informe Anual de Estadísticas Vitales.

Selye, H. (1986). Stress, Cancer and the Mind. In: Day, S.B. (Ed.). Cancer, Stress and Death. New York: Plenum Medical Book Company. 
Niveles de ESTRÉS Y PATRONES DE ADAPTACIÓN...

Taché, J. (1986). Stress as a Cause of Disease. In: Day, S.B. (Ed.). Cancer Stress and Death. New York: Plenum Medical Book Company.

Vega Franki, U. (1992). Ajuste psicosocial de una muestra de pacientes de "cáncer de mama y próstata" hacia la enfermedad. Tesis de maestría inédita, Universidad de Puerto Rico, Recinto de Ciencias Médicas, Escuela Graduada de Salud Pública. 
